\title{
Modelling xenograft tumor growth under antiangiogenic inhibitation with mixed-effects models
}

\author{
Tamás Ferenci*, Johanna Sápi* and Levente Kovács* \\ *Research and Innovation Center of Óbuda University, Physiological Controls Group \\ Óbuda University, Budapest, Hungary \\ Email: \{ferenci.tamas,sapi.johanna,kovacs.levente\}@nik.uni-obuda.hu
}

\begin{abstract}
Antiangiogenic inhibitors offer a promising new treatment modality in oncology. However, the optimal administration regime is often not well-established, despite the fact that it might have substantial impact on the outcome. The aim of the present study was to investigate this issue. Eight weeks old male $\mathrm{C} 57 \mathrm{Bl} / 6$ mice were implanted with $\mathrm{C} 38$ colon adenocarcinoma, and were given either daily $(n=9)$ or single $(n=5)$ dose of bevacizumab; both receiving the same dose the only difference being the administration pattern. Outcome was measured by tracking tumor volume; both caliper and magnetic resonance imaging was employed. Longitudinal growth curves were modelled with mixed-effects models (with correction for autocorrelation and heteroscedasticity, where necessary) to infer on population-level. Several different growth models (exponential, logistic, Gompertz) were applied and compared. Results show that the estimation of the exponential model is very reliable, but it prevents extrapolation in time. Nevertheless, it clearly established the advantage of the continuous regime.
\end{abstract}

\section{INTRODUCTION}

In the clinical practice, there are general protocols for cancer therapies (like chemotherapy, radiotherapy); however, these treatments have a lot of side effects and tumor cells can become resistant to chemotherapy drugs, which makes the usage of new drugs necessary and from the other side increases the treatment cost. Using control engineering methodologies (model identification and controller design), the protocols could become model-based. Model-based design allows to find more effective solutions in healing and individual treatment for the patient.

Tumor growth dynamics can be modeled without therapy and under a certain cancer treatment as well. A promising targeted molecular therapy [1] arose in the last decade is antiangiogenic therapy [2], which aims to stop tumor angiogenesis (i.e. forming new blood vessels), as without a blood supply, tumors cannot grow. A clinically validated tumor growth model under angiogenic inhibition was developed at Harvard University in 1999 by Hahnfeldt et al. [3]. The model describes that reduction of tumor volume based on endothelial reduction. The Hahnfeldt model and its simplified form have been used by most researchers working in the field of antiangiogenic control to design controller and perform simulations. Nevertheless, the Hahnfeldt model has some limitations according to the newest medical research in the field of angiogenic tumor growth [4]. We carried out specific animal experiments to investigate tumor growth under angiogenic inhibition.

In particular, the aim of our study was to determine whether the continuous administration of an antiangiogenic inhibitor offers advantages over the more conservative (higher dose less frequent administration) approach.

\section{Material And Methods}

\section{A. Experimental Settings}

1) Mouse and cancer type: 16 eight weeks old male C57B1/6 mice were implanted with C38 colon adenocarcinoma. A piece of tumor was transplanted subcutaneously in the recipient animal on the 1st day of the experiment.

2) Treatment: The treatment was carried out with bevacizumab which is an exogenous angiogenic drug inhibiting the biological activity of human VEGF [5]. Mice were divided into two groups. Control group ( 5 mice) received bevacizumab in one dose for an 18-day treatment according to the protocol (200 $\mu \mathrm{g}$ bevacizumab with $455 \mu l \quad 0.9 \% \mathrm{NaCl}$ solution) intraperitoneally on the -1 st day and on the 17 th day. Treatment group (9 mice) received one-tenth dose of control dose intraperitoneally spread over 18 days $(1.11 \mu \mathrm{g}$ bevacizumab with $45 \mu l 0.9 \% \mathrm{NaCl}$ solution) every day from the -1 st day of the experiment. The treatment period was 20 days.

3) Tumor volume measurement: Tumor volume was measured with digital caliper and small animal MRI as well. Using digital caliper, two tumor diameters (width, length) can be measured. It can be carried out in vivo during the experiment due to the subcutaneous localization of the tumor. Tumor volume (and the third diameter) has to be approximated, assuming a certain shape for the tumor; due to this, we obtained three different volume measurements based on the caliper measurements, differing according to the applied model. Measurements with caliper were done on the 0th, 2nd, 4 th, 6th, 8th, 10th, 12th, 14th, 16th, 18th and 19th days of the experiment. 


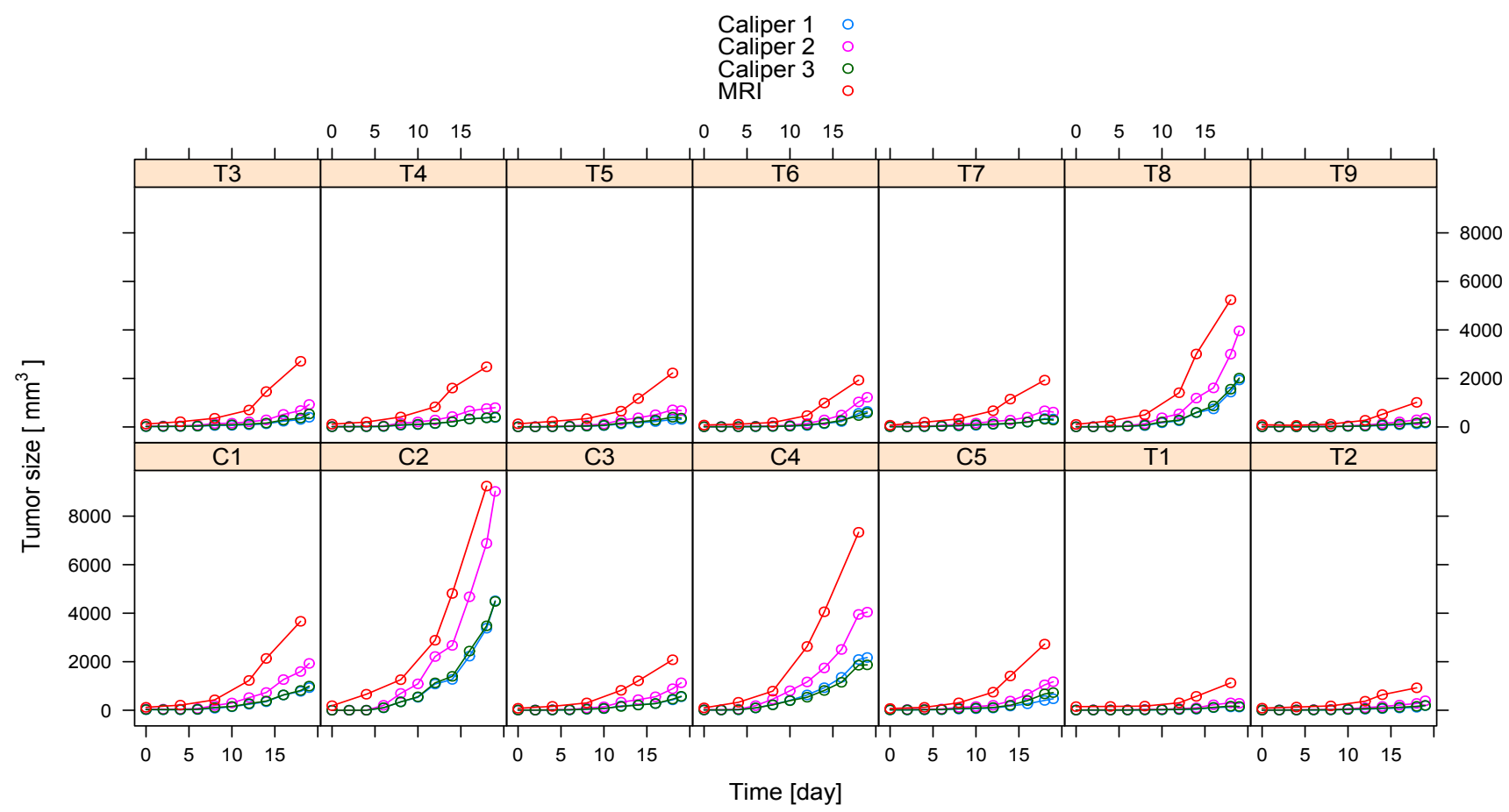

Fig. 1. Measured tumor volumes (with all measurement methods).

Small animal MRI provieds more precise volume measurement. Measurements with small animal MRI were done on the 0th, 4th, 7th, 11th, 14th and 19th days of the experiment.

The measured tumor volumes (with all measurement methods) are shown on Figure 1.

4) Sacrificing mice: All surgery and sacrifice were performed under sodium pentobarbital anaesthesia (Nembutal, $70 \mathrm{mg} / \mathrm{kg}$ ), and all efforts were made to minimize suffering. Mice were monitored daily. Mice were euthanized by $\mathrm{CO}_{2}$ inhalation if tumors contributed to a gain of $>20 \%$ in body weight compared to controls at the same time point. Mice were humanely sacrificed using cervical dislocation.

\section{B. Tumor growth models}

While the growth of tumors involves many complicated biological mechanisms, their overall nature (in terms of weight, size or volume) often follows surprisingly simple patterns. This was recognized decades ago - in particular after the seminal paper of Laird in 1964 [6] - and has been utilized since then both to understand the biological foundations and to provide modelling, for instance in preclinical studies of drug candidates using xenograft tumors implanted in test animals (even though it is known to have certain limitations).

These models might be purely empirical, like the Gompertzian growth discussed by Laird, or they might involve considerations about the underlying biological mechanisms (mechanistic and semi-mechanistic models), like the exponential-linear model by Simeoni [7].

In the present study, empirical models will be applied. While some model (e.g. [8]) directly incorporates the effect of drugs (making them at least semi-mechanistic), the present study will assume that the same - empirical - growth model applies in both the control and the treated group; the drug exerts its effects by modifying the parameters of the curve (see Subsection II-C for details).

1) Exponential growth (inital phase, no plateau): One of the earliest observations about tumor growth modeling was that in many case, the growth - both in vivo and in vitro exhibits exponential nature in its earliest period. Biologically, it correspends to the phase where the resource-limitation is not apparent, and in that sense the tumor can "freely" grow, limited only by its own size which defines the pool of cells that can divide.

Due to this, the growth is governed by the following ordinary differential equation:

$$
\frac{\mathrm{d} V(t)}{\mathrm{d} t}=a V(t)
$$

the solution of which is the well-known exponential growth formula:

$$
V(t)=V_{0} e^{a t},
$$

where $V(t)$ tumor volume (in this case, but can be any size generally), with $V_{0}=V(0)$.

Clearly, this model is only appropriate in the early phase of tumor growth. In particular, it always leads to an infinite growth, the reason being that the effect of the appearence of growth-limiting factors is not accounted for.

As our measurements were made in that early period, even the exponential growth provides adequate fit at first glance. 
Nevertheless, these models are still problematic, even in this case, simply because they offer no possibility to extrapolate in time. Even if the plateau is not yet apparent, we might try to model it using the pre-plateau information - this can not be achieved with exponential model.

Thus, the exponential model will only be used as a reference later on.

2) Sigmoid growth (plateau accounted for): To solve the abovementioned problems, typically sigmoid-like growth curves are assumed. These are able to capture the asymptotical phase, and thus the plateau can be estimated; theoretically even before it starts to appear (albeit with limited reliability for

Two popular from them will be used now, for a review of other possibilities, see [9]-[11].

Note that many such model is a special case of the differential equation (often called the generalized two-parameter model)

$$
\frac{\mathrm{d} V(t)}{\mathrm{d} t}=a V(t)^{\alpha}-b V(t)^{\beta},
$$

with appropriate choice of the $a, \alpha, b, \beta$ parameters. (Actually even the exponential model is a special case with $a=1$, $\alpha=1, b=0$.)

a) Gompertz growth: One of the earliest - and perhaps the most widely used - of the sigmoid models was the Gompertz growth, introduced in 1964 by Laird [6]. It obeys the following differential equation:

$$
\frac{\mathrm{d} V(t)}{\mathrm{d} t}=a V(t)-b V(t) \ln V(t),
$$

giving rise to the Gompertz growth curve:

$$
V(t)=e^{\frac{a}{b}-\left(\frac{a}{b}-\ln V_{0}\right) e^{-b t}} .
$$

(This is also a limiting case of Equation 3 with appropriate definitions [12].)

One well-known property of Gompertz growth is that initial rising period has a higher derivative than the period before reaching the asymptote, i.e. it is not symmetric in the two transitions.

b) Logictic growth: The logistic growth is another wellknown model; in contrast to the Gompertz curve, it is symmetric in both transitions.

It follows the following differential equation:

$$
\frac{\mathrm{d} V(t)}{\mathrm{d} t}=a V(t)-b V(t)^{2}
$$

giving rise to the logistic growth curve:

$$
V(t)=\frac{a / b}{1-\left(1-\frac{a}{b V_{0}}\right) e^{-a t}} .
$$

(This is a special case of Equation 3 with $\alpha=1$ and $\beta=2$.)

\section{Statistical tools}

First, individual curves were fitted for each test animal (using each measurement method) with all three models. Fitting was performed with nonlinear least squares (NLS) approach using the Gauss-Newton algorithm [13], [14].
This provides the best fit as it estimates individual parameters for all subject and measurement method, but this is also the very reason that prevents generalization: all fits are isolated, therefore it is not possible to infer on a higher level (i.e. the population of parameters). As we are now primarily interested not in these particular subjects, but rather on the population from which they are coming, a model will be used which explicitly incorporates this aspect: the mixed effects model [15], [16].

These models assume that parameters are not fixed values, but rather realizations of a random variable, most typically normal random variable, characterized by mean and variance (and possible covariance). Thus the estimation focuses in these parameters.

To formalize: denoting the tumor volume of the $i$ th subject at measurement number $j$ with $V_{i j}$ we have

$$
V_{i j}=f\left(\phi_{i j}, t_{i j}\right)+\varepsilon_{i j}, \quad \varepsilon_{i j} \sim \mathcal{N}\left(0, \sigma^{2}\right),
$$

where $f$ represent the nonlinear functional form - exponential, Gompertz or logistic in this case - of time $\left(t_{i j}\right.$ being the time when the $j$ the measurement was made on the $i$ th subject) determined the parameters $\phi_{i j}$.

Now we assume that these parameters depend on whether the test animal belongs to the treated or to the control group and on the measurement method, that is

$$
\boldsymbol{\phi}_{i j}=\left(\boldsymbol{\beta}_{0}+\mathbf{b}_{i}\right)+\boldsymbol{\beta}_{\text {Group }} \operatorname{Group}_{i}+\boldsymbol{\beta}_{\text {MeasMeth }} \text { MeasMeth }_{i},
$$

where $\mathbf{b}_{i} \sim \mathcal{N}(\mathbf{0}, \boldsymbol{\psi})$. (In this case $\phi_{i j}$ only depends on $i$, in other words, we had no time-varying covariates.) We assumed that $\boldsymbol{\psi}$ is diagonal, i.e. the random effects are uncorrelated.

In other words, we assume that these covariates act by altering the parameters of the - same - functional form. In particular, this means that the effect of drug is incorporated by assuming that the tumor growth obeys the same law under treatment, but with different parameters.

This model assumes that

- the variance of the error terms is a constant (i.e. no heteroscedasticity present),

- the error terms are uncorrelated (i.e. no autocorrelation present).

These assumptions were checked by plotting the standardized residuals versus the fitted values (Figure 2a), and the autocorrelation function of the residuals (Figure $2 b$ ), respectively. In case of violation, appropriate weighting functions and within-subject autocorrelation functions were included in the models (power variance and continuous time AR(1) correlation in the shown example of exponential growth) [15].

Effects are considered significant if $p<0.05$.

\section{Programs used}

R statistical program package [17] (version 3.2.5) was used with the nlme library [18] (version 3.1-127) to carry out the calculations using a custom script developed for this purpose that is available at the corresponding author on request. Visualizations were created with the lattice library [19] (version 0.20-33). 


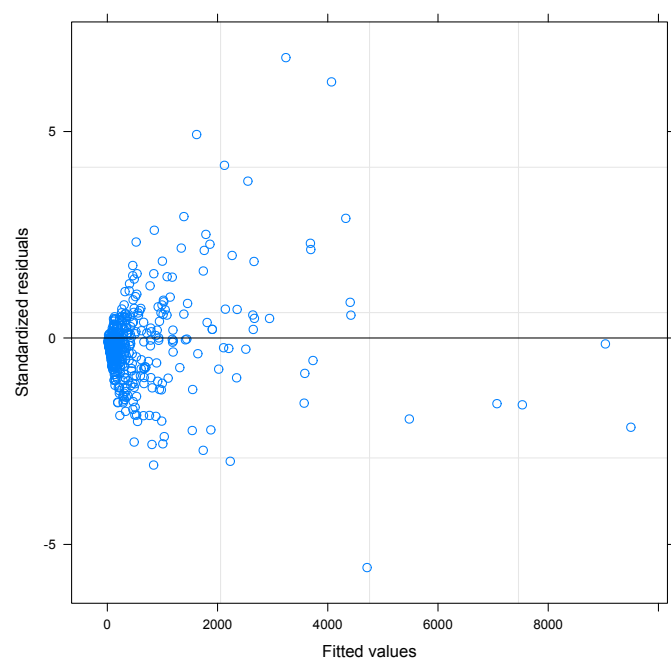

(a) Testing heteroscedasticity

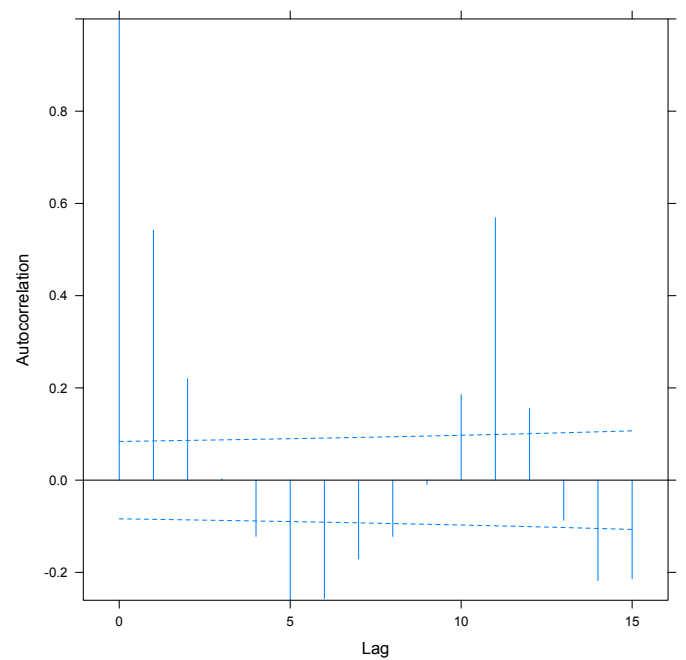

(b) Testing autocorrelation

Fig. 2. Testing the model assumptions for the exponential growth model.

\section{RESULTS}

\section{A. Individual fitting}

Results, displayed as points estimates for the parameters with $95 \%$ confidence intervals are shown on Figure 3 for each subject and measurement method; with each model.

\section{B. Population-level mixed model}

1) Exponential growth: The estimated parameter of the AR(1) correlation was 0.944 , the estimated power in the variance function was 0.952 . With these, the standard deviation of the random effect for the $V_{0}$ was 0.00136 , for the $a$ it was $3.944 \cdot 10^{-7}$. The residual standard deviation was 0.972 , the $A I C$ was 6007 .

In this model, the intercept of $V_{0}$ is 11.9 with MRI measurement having significantly higher values than caliper measurements $(+97.1$ compared to Caliper-1). The intercept of $a$ is 0.268 , but now MRI measurements are having significantly lower $a(-00.561$ compared to Caliper-1).

The treatment's effect is very interesting: it does not significantly alter $V_{0}(p=0.0983)$, but it does have a statistically significant effect on $a$, being associated with -0.0434 change $(p=0.0003)$. I.e. the treatment decreases the rate of growth, but not the initial

2) Sigmoid growth: The results of the mixed models are shown (Figure 4) as - population-level - predicted tumor volumes for Days 0 to 150 (for each measurement type and for the treated/control groups). Note that the original data spanned from Days 0 to 19 , so this exemplifies the extrapolation with the models.

The residual deviation was 86.6 for the logistic fit $(A I C=$ $6600)$, and 660 for the Gompertz fit $(A I C=8670)$.

The effect of treatment was significant for the mid-point parameter of the logistic growth $(p=0.0228)$, but it was not significant for any parameter of the Gompertz growth.

\section{DISCUSSION}

It was possible to reliably estimate all parameters of the exponential growth model, indicated by the consistent and rather narrow confidence intervals in the individual fits and the acceptable residual standard deviation in the mixed model. The results show the effect of the treatment; already demonstrated in an earlier research [20].

In contrast, the estimates for the sigmoid-growth models were exceedingly variable, individual fit was not even possible for some of the cases. The population level model exhibited extremely poor fit, with enormously high residual deviations. In short, it was not possible to reliable estimate these models, the reason being the rather short observation period that was available, showing only the very early period of tumor growth. Nevertheless, results point out the possibility to estimate the plateu phase, which is a very interesting and promosing parameter - in additional to the initial rate of growth, already captured by the exponential model -, but trusthworthy estimation of this requires more observation.

Results also point out the differences between the measurement devices, clearly showing that measurements made with MRI are systematically higher than any of the caliper measurements, no matter which model we used.

One strength of our approach was that it integrates all factors (treatment and measurement device) into one single model, allowing us to investigate both the effect of the treatment and the effect of the applied measuring method at the same time. Also, the mixed effects approach allows a smooth and elegant usage of the individual measurements to create a population-level model. In addition to that, we have the possibility to test several potentional functional forms; with some of them, we are also able to extrapolate in time, and capture clinically relevant parameters. 


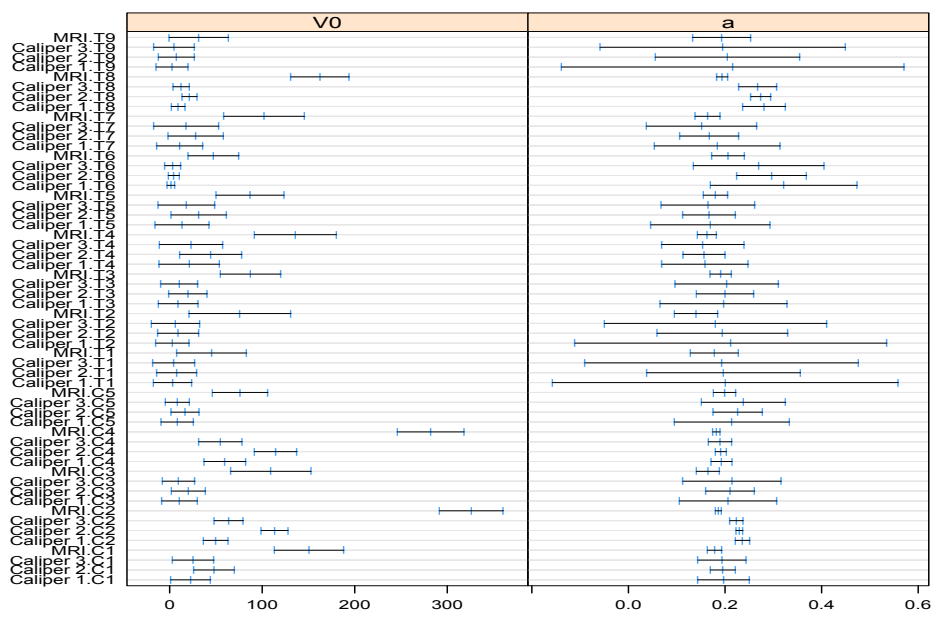

(a) Exponential growth

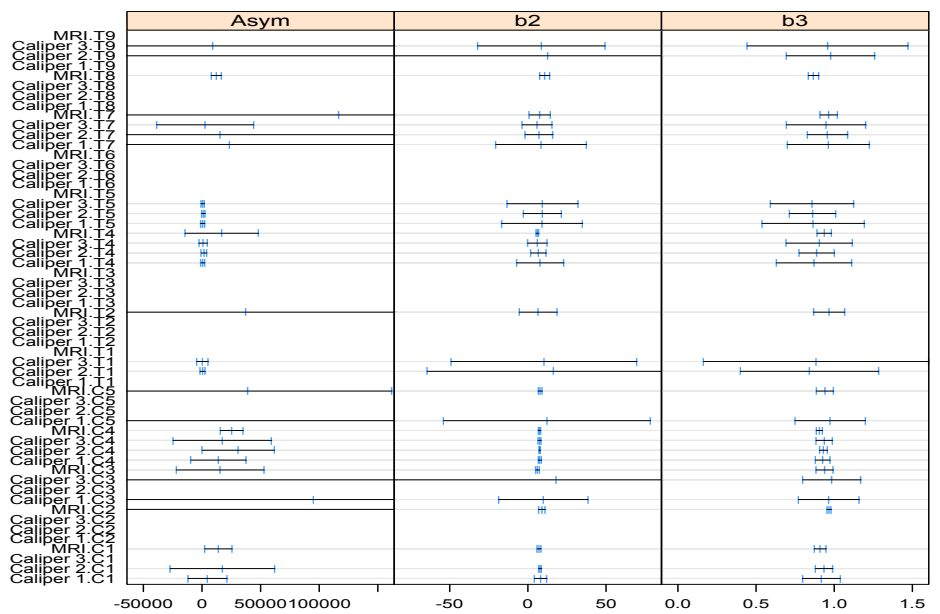

(b) Gompertz growth. Parameters pertain to the following parametrization of the growth function: $V(t)=\mathrm{Asym} \cdot e^{-\mathrm{b} 2 \cdot \mathrm{b} 3^{t}}$.

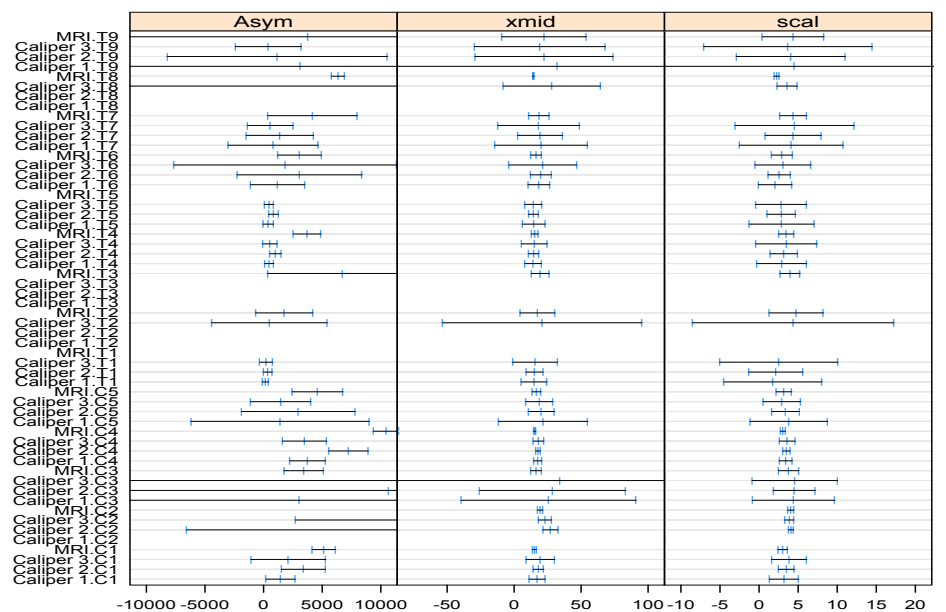

(c) Logistic growth. Parameters pertain to the following parametrization of the growth function: $V(t)=\operatorname{Asym} /\left(1+e^{(\mathrm{xmid}-t) / \mathrm{scal}}\right)$.

Fig. 3. Results of the individual curve fitting (for each animal using each measurement method); with every model. Axis limits were adjusted to show the most important data; extreme values might be cut. Missing estimates indicate cases were NLS was unable to converge. 


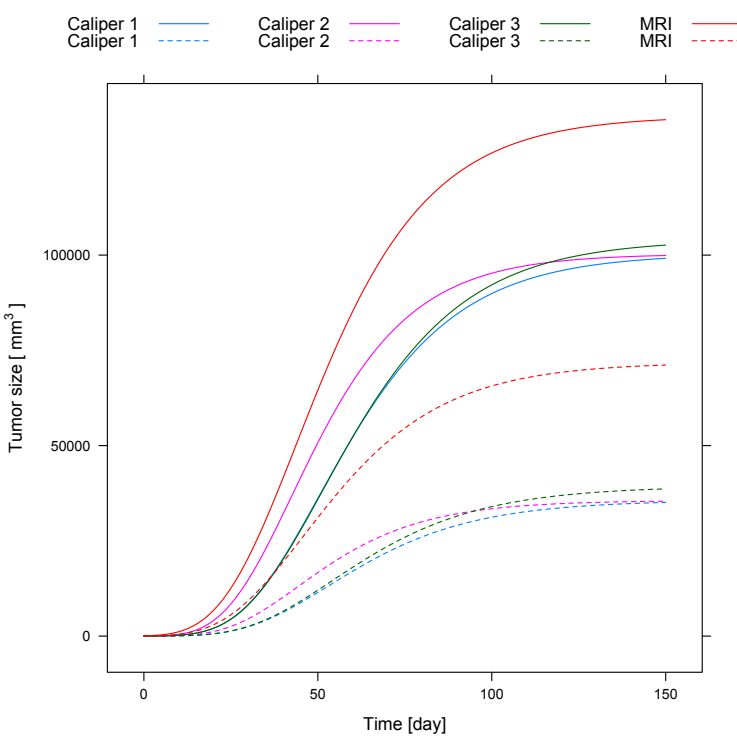

(a) Gompertz growth

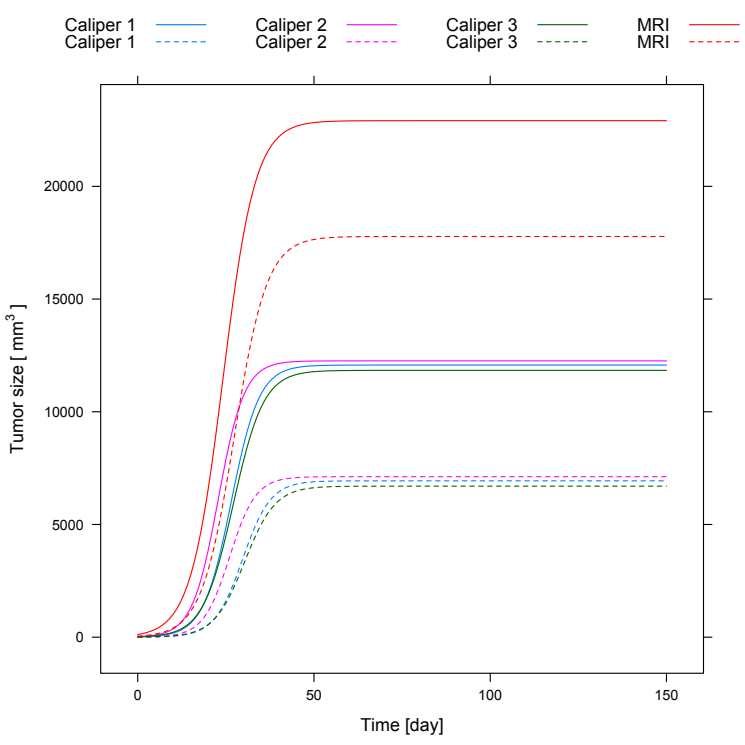

(b) Logistic growth

Fig. 4. Predicted growth (on population-level) of the tumor volume for Days 0 to 150. Different colors indicate different measurement methods (see legend), solid line indicates control group, while dashed line indicates treated group.

The most important limitation was the rather small number of test subjects, and the too short observation period to estimates some of the models.

\section{CONCLUSION}

The exponential model could be estimated in a robust manner, both individually, and in the population-level with the mixed effects model. Results confirm the effect of the treatment, and make it possible to quantify this.

In contrast, the sigmoid-like growth curves were almost impossible to estimate, revealing the limitations of our data. Nevertheless, the possibility to estimate such models - making extrapolation possible - mean a promising opportunity.

\section{ACKNOWLEDGMENT}

The research was supported by the European Research Council Starting Grant ERC-StG 679681.

\section{REFERENCES}

[1] I. Petersen, "Antiangiogenesis, anti-vegf(r) and outlook," in Recent Results In Cancer Research, Targeted Therapies in Cancer, M. Dietel, Ed. Springer - Verlag, 2007.

[2] G. Bergers and L. E. Benjamin, "Tumorigenesis and the angiogenic switch," Nat Rev Cancer, vol. 3(6), pp. 401-410, 2003.

[3] P. Hahnfeldt, D. Panigrahy, J. Folkman, and L. Hlatky, "Tumor development under angiogenic signaling: A dynamical theory of tumor growth, treatment response, and postvascular dormancy," Cancer research, vol. 59, pp. 4770-4775, 1999.

[4] H. Femke and A. W. Griffioen, "Tumour vascularization: sprouting angiogenesis and beyond," Cancer Metastasis Rev, vol. 26(3-4), pp. 489-502., 2007.

[5] European Medicines Agency, "Scientific discussion of Avastin," Available: http://www.ema.europa.eu, 2005, accessed 10 April 2016.

[6] A. K. Laird, "Dynamics of tumour growth," British Journal of Cancer, vol. 18 , no. 3 , p. $490,1964$.
[7] M. Simeoni, P. Magni, C. Cammia, G. De Nicolao, V. Croci, E. Pesenti, M. Germani, I. Poggesi, and M. Rocchetti, "Predictive pharmacokineticpharmacodynamic modeling of tumor growth kinetics in xenograft models after administration of anticancer agents," Cancer Research, vol. 64, no. 3, pp. 1094-1101, 2004.

[8] S. Yamazaki, J. Skaptason, D. Romero, J. H. Lee, H. Y. Zou, J. G. Christensen, J. R. Koup, B. J. Smith, and T. Koudriakova, "Pharmacokineticpharmacodynamic modeling of biomarker response and tumor growth inhibition to an orally available cmet kinase inhibitor in human tumor xenograft mouse models," Drug Metabolism and Disposition, vol. 36, no. 7, pp. 1267-1274, 2008.

[9] P. L. Bonate, "Modeling tumor growth in oncology," in Pharmacokinetics in drug development. Springer, 2011, pp. 1-19.

[10] R. P. Araujo and D. L. S. McElwain, "A history of the study of solid tumour growth: the contribution of mathematical modelling," Bulletin of mathematical biology, vol. 66, no. 5, pp. 1039-1091, 2004.

[11] S. Benzekry, C. Lamont, A. Beheshti, A. Tracz, J. M. Ebos, L. Hlatky, and P. Hahnfeldt, "Classical mathematical models for description and prediction of experimental tumor growth," PLoS Comput Biol, vol. 10, no. 8, p. e1003800, 2014.

[12] M. Marušić, Ž. Bajzer, J. Freyer, and S. Vuk-Pavlović, "Analysis of growth of multicellular tumour spheroids by mathematical models," Cell proliferation, vol. 27, no. 2, pp. 73-94, 1994.

[13] D. M. Bates and D. G. Watts, Nonlinear regression analysis and lts applications. Wiley, 1988.

[14] C. Ritz and J. Streibig, Nonlinear Regression with $R$, ser. Use R! Springer New York, 2008.

[15] J. Pinheiro and D. Bates, Mixed-Effects Models in S and S-PLUS, ser. Statistics and Computing. Springer New York, 2000.

[16] G. Verbeke and G. Molenberghs, Linear Mixed Models for Longitudinal Data, ser. Springer Series in Statistics. Springer New York, 2009.

[17] R Core Team, R: A Language and Environment for Statistical Computing, R Foundation for Statistical Computing, Vienna, Austria, 2016. [Online]. Available: https://www.R-project.org/

[18] J. Pinheiro, D. Bates, S. DebRoy, D. Sarkar, and R Core Team, nlme: Linear and Nonlinear Mixed Effects Models, 2016, r package version 3.1-127. [Online]. Available: http://CRAN.R-project.org/package=nlme

[19] D. Sarkar, Lattice: Multivariate Data Visualization with R. New York: Springer, 2008. [Online]. Available: http://lmdvr.r-forge.r-project.org

[20] J. Sápi, T. Ferenci, D. A. Drexler, and L. Kovács, "Tumor model identification and statistical analysis," in Systems, Man, and Cybernetics (SMC), 2015 IEEE International Conference on, 2015, pp. 2481-2486. 